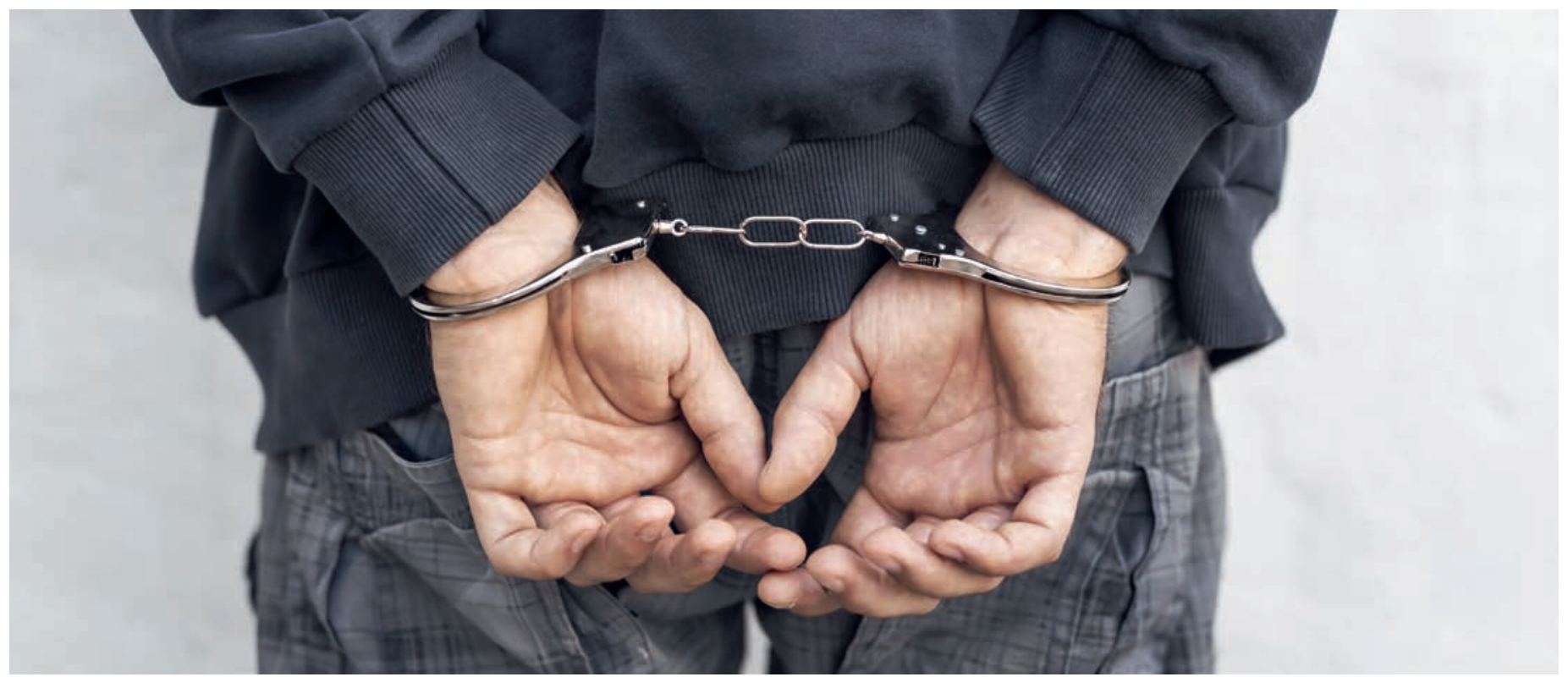

\title{
Betreuung von Personen mit mutmasslichem Bodypacking
}

\section{Hans Wolffa, Michelle Salathéb, Yvonne Gillic}

a Prof. Dr. med., Mitglied der Zentralen Ethikkommission SAMW (ZEK), Vizepräsident der Konferenz Schweiz. Gefängnisärzte (KSG)

${ }^{b}$ lic. iur. MAE, stv. Generalsekretärin SAMW, Leiterin Ressort Ethik

c Dr. med., Mitglied der Zentralen Ethikkommission SAMW (ZEK), Mitglied Zentralvorstand FMH

1 Differenziert wird zwischen Bodypacking, Bodystuffing und Bodypushing. Bodypacking bezeichnet das Schlucken mehrerer Drogenpäckchen, oft bis zu 100 Stück. Beim Bodystuffing werden die Drogen oft unmittelbar vor einer Kontrolle verschluckt, um sie vor den Beamten zu verbergen. Beim Bodypushing werden die Drogen vaginal oder rektal versteckt.

Ärztinnen und Ärzte, die Personen mit mutmasslichem Bodypacking betreuen, sind mit widersprüchlichen Erwartungen konfrontiert. In Absprache mit der Konferenz Schweiz. Gefängnisärzte (KSG) hat die Zentrale Ethikkommission der SAMW die Richtlinien «Ausübung der ärztlichen Tätigkeit bei inhaftierten Personen» mit einem Anhang ergänzt, der die Aufgaben der involvierten Ärztinnen und Ärzte klärt.

Personen, die Drogen in ihrem Körper illegal über die Grenze transportieren, gehen ein hohes gesundheitliches Risiko ein. Die Drogen werden als Päckchen, meist Kokain portioniert zu 10 bis 20 Gramm (sog. Bodypacks ${ }^{1}$ ), geschluckt. Wenn ein solches Bodypack platzt, besteht unmittelbare Lebensgefahr. Fällt eine Person mit mutmasslichem Bodypack an der Grenze auf, wird der Verdacht im Spital mittels Computertomogramm (CT) abgeklärt. Die verdächtigte Person kann diese Untersuchung verweigern, weil das CT eine Strahlen- belastung darstellt. Eine solche Weigerung muss respektiert werden. Eine Alternative zum CT ist die Überwachung der Ausscheidungen bis zur Erhärtung oder Widerlegung des Verdachts.

Bei einer solchen Verdachtsabklärung entsteht für die involvierten Ärztinnen und Ärzte oftmals ein Rollenkonflikt: Sie müssen sowohl den Verdacht «Bodypacking» abklären als auch die betroffenen Personen gesundheitlich überwachen, um eine sichere Ausscheidung der gefährlichen Fracht zu gewährleisten. Die Ärz- 
tinnen und Ärzte können jedoch nicht gleichzeitig eine Experten- und eine Therapeutenrolle wahrnehmen.

\section{Kritik durch Nationale Kommission zur Verhütung von Folter}

Am 3. April 2017 wandten sich Ärzte und Ärztinnen des Spitalzentrums Oberwallis an das Generalsekretariat der SAMW und baten die Zentrale Ethikkommission (ZEK) um eine Stellungnahme zum Umgang mit verdächtigten «Bodypackerinnen». Hintergrund dieser Anfrage war, dass die Assistenzärzte des Spitalzentrums Oberwallis von der Grenzwacht angewiesen worden waren, bei allen verdächtigen Personen bildgebende Verfahren (CTs) durchzuführen. Die Anordnung erfolgte, ohne dass die beauftragten Ärzte einen Entscheidungsspielraum hatten. Sie wurden zudem nicht informiert, ob und wie die verdächtigten Personen über das Vorgehen aufgeklärt worden waren.

\section{Die Ärztinnen und Ärzte können nicht gleich-} zeitig eine Experten- und eine Therapeutenrolle wahrnehmen.

Die Nationale Kommission zur Verhütung von Folter veröffentlichte 2018 einen Bericht, in dem sie eine übermässige Strahlenbelastung der verdächtigten Personen durch das Vorgehen der Walliser Grenzpolizei kritisierte. ${ }^{2}$ Zudem waren nur 9 Prozent der bei verdächtigen Personen durchgeführten CT-Untersuchungen positiv, d.h., 91 Prozent der untersuchten Personen wurden einer unnötigen Strahlenbelastung ausgesetzt. Darüber hinaus sollen Untersuchungen bei schwangeren Frauen durchgeführt worden sein, ungeachtet der Risiken für das ungeborene Kind. Der
Bericht hielt weiter fest, dass Kliniken in mehreren Kantonen die Möglichkeit vorsehen, radiologische Untersuchungen auch dann durchzuführen, wenn sich die verdächtigte Person dieser widersetzt.

Aus medizin-ethischer Sicht sind die beschriebenen Umstände inakzeptabel. Personen mit mutmasslichem Bodypacking, die sich keiner Strahlenbelastung aussetzen möchten, können medizinisch überwacht

\section{Nur 9 Prozent der bei verdächtigen Personen} durchgeführten CT-Untersuchungen waren positiv.

werden, ohne dass ein CT verordnet und unter Zwang durchgeführt wird. Der neue Anhang H der SAMWRichtlinien "Ausübung der ärztlichen Tätigkeit bei inhaftierten Personen» bietet hierzu Hilfestellungen an. Er nimmt die für die Praxis notwendige Rollenklärung der involvierten Ärztinnen und Ärzte vor, indem er zwischen "Expertenaufgaben» (Nachweis von Bodypacks) und "therapeutischen Aufgaben" (Überwachung, medizinische Intervention) trennt. Zudem werden wichtige anamnestische und therapeutische Aspekte bei asymptomatischen und symptomatischen Patienten beschrieben. Der Anhang verweist auf das erhöhte Suizidrisiko, das in diesen Situationen besteht, und die Notwendigkeit der medizinischen Überwachung.

Die SAMW empfiehlt den Schweizer Spitälern und weiteren Institutionen, die mit Bodypacking befasst sind, die ergänzten medizin-ethischen Richtlinien anzuwenden. Die Richtlinien und der neue Anhang H können auf der SAMW-Website heruntergeladen werden: samw.ch/bodypacking.

Bildnachweis

(c) Vladans | Dreamstime.com (Symbolbild) 